\title{
LIST OF REVIEWERS
}

The Editorial Board of the Journal of Nutritional Science would like to thank the following for their contribution as peer reviewers in 2021:

\author{
Laxman Acharya \\ Jon (J.D.) Adams \\ Folasade Adebayo \\ Pooyan Afzali Harsini \\ Puja Agarwal \\ Bruno Akpakpo \\ Fahmida Akter \\ Ghadeer Aljuraiban \\ Margaret Allman-Farinelli \\ Cláudia Almeida \\ Haleemah Alsabah \\ Cristian Alvarez \\ Ramya Ambikapathi \\ Lucilene Anastácio \\ Donato Angelino \\ Prawej Ansari \\ Luciene Araújo \\ Giannis Arnaoutis \\ Omar Elind Arroyo-Helguera \\ Elaheh Asgari \\ Dagfinn Aune \\ Jessica Ayensu \\ Yeji Baek \\ Yu Bai \\ Anne Marie Bakke \\ Jesse Bakke \\ Rodrigo Bancalari \\ Costas Bardis \\ Guillermina Barril-Cuadrado \\ Laurent Beghin \\ Martha Belury \\ Mariela Bernabe-Garcia \\ Julia Bird \\ Rosa Maria Borzì \\ Lana Braudes Silva
}

Jessica Briffa
Grant Brinkworth
Nidhi Budhalakoti
Yingting (Tina) Cao
Lucy Capelle
Carla Carilho
Juan Carmona-Hernandez
Jennifer Carter
Prasanth Chelikani
Anna Cherta Murillo
Tsuyoshi Chiba
Miriam Clegg
Marie Conway
Alfredo Jorge Costa Teixeira
Vassiliki Costarelli
Peter Curtis
Kássia da Silva
Monica de Assunção
Johan de Vogel-van den Bosch
David Doledec
Theogene Dusingizimana
Rejun Fang
Awat Feizi
Elisa Felix-Soriano
Artur Ferron
Sondos Flieh
Joy Nolte Fong
Bartosz Fotschki
Fabiane Francisqueti
Cara Frankenfeld
David Frankenfield
Patrícia Freitas
Sanjeev Galande
Michael Georgoulis
Dereje Getahun

Neda Gilani
Rubia Giordani
Andrea Glenn
William Grant
Paul Hager
Masahide Hamaguchi
Osama Hamdy
Akinori Hara
Rachel Harris
Christina Hartmann
Ashleigh Haynes
Pingli He
Maria Hegelund
Oscar Herran
Marion Hetherington
Daniel Hinojosa-Nogueira
Kelly Hirko
Gladys Honein-AbouHaidar
Min Kyaw Htet
Katelin Hudak
Crissy Irani
Francois Iris
Samira Jafari
Hao Jing
Manuela Jomori
Tony Jose
Shinyoung Jun
JungHee Kang
Merve Şeyda Karaçil Ermumcu
Nena Karavasiloglou
Therese Karlsson
Ryoko Katagiri
Foorough Kavian
Aparna Kohli
Yoko Komada

(C) The Author(s), 2022. Published by Cambridge University Press on behalf of The Nutrition Society. This is an Open Access article, distributed under the terms of the Creative Commons Attribution-NonCommercial-NoDerivatives licence (https://creativecommons.org/licenses/by-nc-nd/4.0/), which permits non-commercial re-use, distribution, and reproduction in any medium, provided the original work is unaltered and is properly cited. The written permission of Cambridge University Press must be obtained for commercial re-use or in order to create a derivative work. 
Aruna Jyothi Kora

Rena Kosti

Ahou Nadege Charlotte Kouadio N'gbesso

Solo Kuvibidila

Abera Lambebo

Brenda Leung

Xilong Li

Siew Lim

Junhua Liu

Su Peng Loh

Esther Lopez-Garcia

Fernanda Madruga

Simonette Mallard

François Mariotti

Y. Fabiola Marquez-Sandoval

Monica Mars

Kate Maslin

Maria Gabriela Matias de Pinho

Mai Matsumoto

Nirupa Matthan

Elaine McCarthy

Anine Medin

Samson Mideksa

Lorenza Mistura

Arlene Mitchell

Violeta Moya-Alvarez

Patrick Mullie
Chisato Nagata

Amanuel Nana

Elizabeth Neale

Michael Nelson

Maku Ocansey

Prachi Pandit

Maria Papageorgiou

Indira Paz-Graniel

Leta Pilic

Sally Poppitt

Sarah Purcell

Qonita Rachmah

Kathy Redfern

Gail Rees

Raylene Reimer

Lindsay Robinson

Ana Rodriguez-Mateos

Adriana Jheny Rodriguez-Mendez

Sabine Rohrmann

Mahama Saaka

Ferguson Saapiire

Mehdi Sadeghian

Isabela Sattamini

Shabihul Sayed

Pramith Senaratne

Leila Setayesh

Nitin Shah
Nana Shinozaki

Anne Sidnell

Dayse Silva

Jessica Smith

Robin Spiller

Catherine Steele

Lijuan Sun

Zhihong Sun

Andrew Thorne-Lyman

Isis Trevenzoli

Kirstin Vach

Vincent van Buul

Natalia Vázquez-Manjarrez

Alvaro Vigo

Elena Villacrés

Roberto Volpe

Wendy Walwyn

Leigh Ward

Sarah Warkentin

Patrícia Weidlich

Heidi Wengreen

Jie Yin

Katsuhiko Yokoi

Xiaoyi Yuan

Raul Zamora-Ros

Jing Zhang

Stanley Zlotkin 